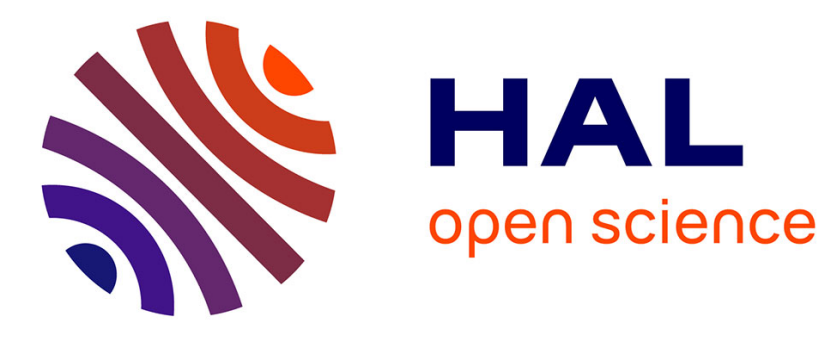

\title{
Nitsche-based finite element method for contact with Coulomb friction
}

\author{
Franz Chouly, Patrick Hild, Vanessa Lleras, Yves Renard
}

\section{To cite this version:}

Franz Chouly, Patrick Hild, Vanessa Lleras, Yves Renard. Nitsche-based finite element method for contact with Coulomb friction. European Conference on Numerical Mathematics and Advanced Applications ENUMATH 2017, University of Bergen, Sep 2017, Voss, Norway. pp.839-847, 10.1007/9783-319-96415-7_79. hal-01654487

\section{HAL Id: hal-01654487 \\ https://hal.science/hal-01654487}

Submitted on 4 Dec 2017

HAL is a multi-disciplinary open access archive for the deposit and dissemination of scientific research documents, whether they are published or not. The documents may come from teaching and research institutions in France or abroad, or from public or private research centers.
L'archive ouverte pluridisciplinaire HAL, est destinée au dépôt et à la diffusion de documents scientifiques de niveau recherche, publiés ou non, émanant des établissements d'enseignement et de recherche français ou étrangers, des laboratoires publics ou privés. 


\title{
Nitsche-based finite element method for contact with Coulomb friction
}

\author{
F. Chouly ${ }^{1}$, P. Hild ${ }^{2}$, V. Lleras ${ }^{3}$ and Y. Renard ${ }^{4}$ \\ 1 Université de Franche Comté, 16 route de Gray, 25000 Besançon, \\ franz.chouly@univ-fcomte.fr \\ 2 Institut de Mathématiques de Toulouse ; UMR 5219. Université de Toulouse ; \\ CNRS. UPS-IMT, F-31062 Toulouse Cedex 9, France, \\ phild@math.univ-toulouse.fr \\ 3 IMAG, Univ Montpellier, CNRS, Place Eugène Bataillon, 34090 Montpellier, \\ France, vanessa.lleras@umontpellier.fr \\ 4 INSA de Lyon, 20 Avenue Albert Einstein, 69100 Villeurbanne, \\ Yves.Renard@insa-lyon.fr
}

\begin{abstract}
The aim of this paper is to provide some mathematical results for the discrete problem associated to contact with Coulomb friction, in linear elasticity, when finite elements and Nitsche method are considered. We consider both static and dynamic situations. We establish existence and uniqueness results under appropriate assumptions on physical (friction coefficient) and numerical parameters. These results are complemented by a numerical assessment of convergence.
\end{abstract}

Many problems involve frictional contact, and are approximated numerically using The Finite Element Method. In this paper we deal with the Nitsche method originally proposed in [17] and that aims at treating the boundary or interface conditions in a weak sense, thanks to a consistent penalty term. It differs in this aspect from standard penalization techniques and from mixed methods since no Lagrange multiplier is needed and no discrete inf sup condition must be fullfilled. A Nitsche-based FEM has been proposed and analyzed for static frictionless unilateral contact in [6,9], and extended to dynamic contact in $[7,8]$. Very few works deal with the adaptation of Nitsche's method to frictional contact (see [5] where recent achievements in applying Nitsche's method to some contact and friction problems are summarized): the Tresca's friction problem is only considered in $[4,11]$ and numerical results for Coulomb friction are presented in $[18,20]$.

In this paper we are interested in some existence and uniqueness results at the discrete level in statics and dynamics. For the continuous static friction problem, existence of solutions hold when the friction coefficient is small enough [13]. In [19], a uniqueness result has been obtained with the assumption that a regular solution exists and that the friction coefficient is sufficiently small. At the discrete level, difficulties appear in the numerical analysis of the method [14]. Results of well-posedness for frictional contact in the dynamic case are presented in [16] for a normal compliance model, in $[1,2]$ for discrete systems of particles and in $[12,15]$ for the modified mass method. 


\section{Setting and discretization}

We consider an elastic body $\Omega$ in $\mathbb{R}^{d}$ with $d=2,3$. Small strain assumptions are made. The boundary $\partial \Omega$ of $\Omega$ is polygonal $(d=2)$ or polyhedral $(d=3)$. The outward unit normal vector on $\partial \Omega$ is denoted $\mathbf{n}$. We suppose that $\partial \Omega$ consists in three nonoverlapping parts $\Gamma_{D}$ on which the body is clamped, $\Gamma_{N}$ and the contact boundary $\Gamma_{C}$, with meas $\left(\Gamma_{D}\right)>0$ and meas $\left(\Gamma_{C}\right)>0$. The contact boundary is supposed to be a straight line segment when $d=2$ or a polygon when $d=3$ to simplify. In the reference configuration, the body is in frictional contact on $\Gamma_{C}$ with a rigid foundation and we suppose that the unknown contact zone during deformation is included into $\Gamma_{C}$. It is subjected to volume forces $\mathbf{f}$ in $\Omega$ and to surface loads $\mathbf{g}$ on $\Gamma_{N}$.

Static problem We consider the unilateral contact problem with Coulomb friction in linear elastostatics. It consists in finding the displacement field $\mathbf{u}: \Omega \rightarrow \mathbb{R}^{d}$ verifying the equations and conditions (1)-(2):

$$
\begin{aligned}
& \operatorname{div} \boldsymbol{\sigma}(\mathbf{u})+\mathbf{f}=\mathbf{0} \quad \text { in } \Omega, \quad \boldsymbol{\sigma}(\mathbf{u})=\mathbf{A} \varepsilon(\mathbf{u}) \quad \text { in } \Omega, \\
& \mathbf{u}=\mathbf{0} \quad \text { on } \Gamma_{D}, \quad \boldsymbol{\sigma}(\mathbf{u}) \mathbf{n}=\mathbf{g} \quad \text { on } \Gamma_{N},
\end{aligned}
$$

The conditions defining unilateral contact with Coulomb friction on $\Gamma_{C}$ are:

$$
\begin{gathered}
u_{n} \leq 0, \quad \sigma_{n}(\mathbf{u}) \leq 0, \quad \sigma_{n}(\mathbf{u}) u_{n}=0 \\
\mathbf{u}_{\mathbf{t}}=\mathbf{0} \quad \Longrightarrow\left|\boldsymbol{\sigma}_{\mathbf{t}}(\mathbf{u})\right| \leq-\mathscr{F} \sigma_{n}(\mathbf{u}) \\
\mathbf{u}_{\mathbf{t}} \neq \mathbf{0} \quad \Longrightarrow \boldsymbol{\sigma}_{\mathbf{t}}(\mathbf{u})=\mathscr{F} \sigma_{n}(\mathbf{u}) \frac{\mathbf{u}_{\mathbf{t}}}{\left|\mathbf{u}_{\mathbf{t}}\right|}
\end{gathered}
$$

where $\mathscr{F} \geq 0$ stands for the friction coefficient. The notation $\boldsymbol{\sigma}=\left(\sigma_{i j}\right), 1 \leq$ $i, j \leq d$, stands for the stress tensor field, $\varepsilon(\mathbf{v})=\left(\boldsymbol{\nabla} \mathbf{v}+\boldsymbol{\nabla} \mathbf{v}^{T}\right) / 2$ represents the linearized strain tensor field and $\mathbf{A}$ is the fourth order symmetric elasticity tensor having the usual uniform ellipticity and boundedness property.

Dynamic problem We consider the unilateral contact problem with Coulomb friction in linear elastodynamics during a time interval $[0, T)$ where $T>0$ is the final time. We denote by $\Omega_{T}:=(0, T) \times \Omega$ the time-space domain, and similarly $\Gamma_{D T}:=(0, T) \times \Gamma_{D}, \Gamma_{N T}:=(0, T) \times \Gamma_{N}$ and $\Gamma_{C T}:=(0, T) \times \Gamma_{C}$. We note $\dot{\mathbf{u}}$ the velocity of the elastic body and $\ddot{\mathbf{u}}$ its acceleration; $\mathbf{u}_{0}$ is the initial displacement and $\dot{\mathbf{u}}_{0}$ is the initial velocity. The density of the elastic material is denoted by $\rho$ and is supposed to be a constant. The problem then consists in finding the displacement field $\mathbf{u}:[0, T) \times \Omega \rightarrow \mathbb{R}^{d}$ verifying the equations and conditions (3)-(4):

$$
\begin{aligned}
\rho \ddot{\mathbf{u}}-\operatorname{div} \boldsymbol{\sigma}(\mathbf{u})=\mathbf{f} & \text { in } \Omega_{T}, & \boldsymbol{\sigma}(\mathbf{u}) & =\mathbf{A} \varepsilon(\mathbf{u}) & & \text { in } \Omega_{T}, \\
\mathbf{u}=\mathbf{0} & \text { on } \Gamma_{D T}, & \boldsymbol{\sigma}(\mathbf{u}) \mathbf{n} & =\mathbf{g} & & \text { on } \Gamma_{N T}, \\
\mathbf{u}(0, \cdot)=\mathbf{u}_{0} & \text { in } \Omega, & \dot{\mathbf{u}}(0, \cdot) & =\dot{\mathbf{u}}_{0} & & \text { in } \Omega,
\end{aligned}
$$


The conditions defining unilateral contact with Coulomb friction on $\Gamma_{C T}$ are:

$$
\begin{aligned}
& u_{n} \leq 0, \quad \sigma_{n}(\mathbf{u}) \leq 0, \quad \sigma_{n}(\mathbf{u}) u_{n}=0 \\
& \dot{\mathbf{u}}_{\mathbf{t}}=\mathbf{0} \quad \Longrightarrow\left|\boldsymbol{\sigma}_{\mathbf{t}}(\mathbf{u})\right| \leq-\mathscr{F} \sigma_{n}(\mathbf{u}) \\
& \dot{\mathbf{u}}_{\mathbf{t}} \neq \mathbf{0} \quad \Longrightarrow \boldsymbol{\sigma}_{\mathbf{t}}(\mathbf{u})=\mathscr{F} \sigma_{n}(\mathbf{u}) \frac{\dot{\mathbf{u}}_{\mathbf{t}}}{\left|\dot{\mathbf{u}}_{\mathbf{t}}\right|}
\end{aligned}
$$

Additionally the initial displacement $\mathbf{u}_{0}$ should satisfy the compatibility condition $u_{0 n} \leq 0$ on $\Gamma_{C}$.

$$
\begin{aligned}
& \text { Proposition 1. Let } \gamma \text { be a positive function defined on } \Gamma_{C} \text {. } \\
& \text { Static case: The frictional contact conditions (2) can be reformulated as } \\
& \text { follows: } \\
& \qquad \sigma_{n}(\mathbf{u})=\left[\sigma_{n}(\mathbf{u})-\gamma u_{n}\right]_{\mathbb{R}^{-}}, \boldsymbol{\sigma}_{\mathbf{t}}(\mathbf{u})=\left[\boldsymbol{\sigma}_{\mathbf{t}}(\mathbf{u})-\gamma \mathbf{u}_{\mathbf{t}}\right]_{\left(-\mathscr{F}\left[\sigma_{n}(\mathbf{u})-\gamma u_{n}\right]_{\mathbb{R}^{-}}\right)} \text {. } \\
& \text { Dynamic case: The frictional contact conditions (4) on } \Gamma_{C T} \text { are equivalent } \\
& \text { to: } \\
& \left.\qquad \sigma_{n}(\mathbf{u})=\left[\sigma_{n}(\mathbf{u})-\gamma u_{n}\right]_{\mathbb{R}^{-}}, \boldsymbol{\sigma}_{\mathbf{t}}(\mathbf{u})=\left[\boldsymbol{\sigma}_{\mathbf{t}}(\mathbf{u})-\gamma \dot{\mathbf{u}}_{\mathbf{t}}\right]_{\left(-\mathscr{F}\left[\sigma_{n}(\mathbf{u})-\gamma u_{n}\right]_{\mathbb{R}^{-}}\right.}\right)
\end{aligned}
$$

The notation $[\cdot]_{\mathbb{R}^{-}}$stands for the projection onto $\mathbb{R}^{-}\left([x]_{\mathbb{R}^{-}}=\frac{1}{2}(x-|x|)\right.$ for $x \in \mathbb{R})$. Moreover, for any $\alpha \in \mathbb{R}^{+}$, we introduce the notation $[\cdot]_{\alpha}$ for the orthogonal projection onto $\mathscr{B}(\mathbf{0}, \alpha) \subset \mathbb{R}^{d-1}$, where $\mathscr{B}(\mathbf{0}, \alpha)$ is the closed ball centered at the origin $\mathbf{0}$ and of radius $\alpha .\|\cdot\|_{s, D}=(\cdot, \cdot)_{s, D}^{\frac{1}{2}}$ denotes the norm of $\left(H^{s}(D)\right)^{d}$.

Let $\mathbf{V}^{h} \subset \mathbf{V}:=\left\{\mathbf{v} \in\left(H^{1}(\Omega)\right)^{d}: \mathbf{v}=\mathbf{0}\right.$ on $\left.\Gamma_{D}\right\}$, be a family of finite dimensional vector spaces indexed by $h$ coming from a family $\mathcal{T}^{h}$ of triangulations of the domain $\Omega$ supposed to be regular and quasi-uniform. We choose a standard Lagrange finite element method of degree $k$ with $k=1$ or $k=2$, i.e.:

$$
\mathbf{V}^{h}=\left\{\mathbf{v}^{h} \in\left(\mathscr{C}^{0}(\bar{\Omega})\right)^{d}:\left.\mathbf{v}^{h}\right|_{K} \in\left(P_{k}(K)\right)^{d}, \forall K \in \mathcal{T}^{h}, \mathbf{v}^{h}=\mathbf{0} \text { on } \Gamma_{D}\right\} .
$$

We consider in what follows that $\gamma=\gamma_{h}$ is a positive piecewise constant function on the contact interface $\Gamma_{C}$ which satisfies $\left.\gamma\right|_{K \cap \Gamma_{C}}=\gamma_{0} h_{K}$, for every $K$ that has a non-empty intersection of dimension $d-1$ with $\Gamma_{C}$, and where $\gamma_{0}$ is a positive given constant.

Let us define the discrete linear operators for a fixed parameter $\Theta \in \mathbb{R}$

$$
\mathrm{P}_{\Theta, \gamma}^{\mathbf{n}}: \begin{aligned}
& \mathbf{V}^{h} \rightarrow \quad L^{2}\left(\Gamma_{C}\right) \\
& \mathbf{v}^{h} \mapsto \Theta \sigma_{n}\left(\mathbf{v}^{h}\right)-\gamma v_{n}^{h},
\end{aligned} \quad \mathbf{P}_{\Theta, \gamma}^{\mathbf{t}}: \begin{aligned}
& \mathbf{V}^{h} \rightarrow\left(L^{2}\left(\Gamma_{C}\right)\right)^{d-1} \\
& \mathbf{v}^{h} \mapsto \Theta \boldsymbol{\sigma}_{t}\left(\mathbf{v}^{h}\right)-\gamma \mathbf{v}_{\mathbf{t}}^{h},
\end{aligned}
$$




$$
Q_{\gamma}^{\mathbf{t}:} \begin{gathered}
\mathbf{V}^{h} \times \mathbf{V}^{h} \rightarrow\left(L^{2}\left(\Gamma_{C}\right)\right)^{d-1} \\
\left(\mathbf{v}^{h}, \dot{\mathbf{v}}^{h}\right) \mapsto \boldsymbol{\sigma}_{\mathbf{t}}\left(\mathbf{v}^{h}\right)-\gamma \dot{\mathbf{v}}_{\mathbf{t}}^{h},
\end{gathered} \quad L\left(\mathbf{v}_{h}\right)=\int_{\Omega} \mathbf{f} \cdot \mathbf{v}_{h} d \Omega+\int_{\Gamma_{N}} \mathbf{g} \cdot \mathbf{v}_{h} d \Gamma,
$$

and the bilinear form:

$$
A_{\Theta \gamma}\left(\mathbf{u}^{h}, \mathbf{v}^{h}\right)=\int_{\Omega} \boldsymbol{\sigma}\left(\mathbf{u}^{h}\right): \varepsilon\left(\mathbf{v}^{h}\right) d \Omega-\int_{\Gamma_{C}} \frac{\Theta}{\gamma} \boldsymbol{\sigma}\left(\mathbf{u}^{h}\right) \mathbf{n} \cdot \boldsymbol{\sigma}\left(\mathbf{v}^{h}\right) \mathbf{n} d \Gamma .
$$

Discrete static problem: The Nitsche-based formulation for unilateral contact with Coulomb friction reads :

$$
\left\{\begin{array}{l}
\text { Find } \mathbf{u}^{h} \in \mathbf{V}^{h} \text { such that: } \forall \mathbf{v}^{h} \in \mathbf{V}^{h} \\
A_{\Theta \gamma}\left(\mathbf{u}^{h}, \mathbf{v}^{h}\right)+\int_{\Gamma_{C}} \frac{1}{\gamma}\left[\mathrm{P}_{1, \gamma}^{\mathbf{n}}\left(\mathbf{u}^{h}\right)\right]_{\mathbb{R}^{-}} \mathrm{P}_{\Theta, \gamma}^{\mathbf{n}}\left(\mathbf{v}^{h}\right) d \Gamma \\
+\int_{\Gamma_{C}} \frac{1}{\gamma}\left[\mathbf{P}_{1, \gamma}^{\mathbf{t}}\left(\mathbf{u}^{h}\right)\right]_{\left(-\mathscr{F}\left[\mathrm{P}_{1, \gamma}^{\mathbf{n}}\left(\mathbf{u}^{h}\right)\right]_{\mathbb{R}^{-}}\right)} \cdot \mathbf{P}_{\Theta, \gamma}^{\mathbf{t}}\left(\mathbf{v}^{h}\right) d \Gamma=L\left(\mathbf{v}^{h}\right) .
\end{array}\right.
$$

Semi discretized dynamic problem: Our space semi-discretized Nitschebased method for frictional unilateral contact problems in elastodynamics then reads:

$$
\left\{\begin{array}{l}
\text { Find } \mathbf{u}^{h}:[0, T] \rightarrow \mathbf{V}^{h} \text { such that for } t \in[0, T]: \forall \mathbf{v}^{h} \in \mathbf{V}^{h} \\
\left\langle\rho \ddot{\mathbf{u}}^{h}(t), \mathbf{v}^{h}\right\rangle+A_{\Theta \gamma}\left(\mathbf{u}^{h}(t), \mathbf{v}^{h}\right)+\int_{\Gamma_{C}} \frac{1}{\gamma}\left[\mathrm{P}_{1, \gamma}^{\mathbf{n}}\left(\mathbf{u}^{h}(t)\right)\right]_{\mathbb{R}^{-}} \mathrm{P}_{\Theta, \gamma}^{\mathbf{n}}\left(\mathbf{v}^{h}\right) d \Gamma \\
+\int_{\Gamma_{C}} \frac{1}{\gamma}\left[Q_{\gamma}^{\mathbf{t}}\left(\mathbf{u}^{h}(t), \dot{\mathbf{u}}^{h}(t)\right)\right]\left(-\mathscr{F}\left[\mathrm{P}_{1, \gamma}^{\mathbf{n}}\left(\mathbf{u}^{h}(t)\right)\right]_{\mathbb{R}^{-}}\right) \cdot \mathbf{P}_{\Theta, \gamma}^{\mathbf{t}}\left(\mathbf{v}^{h}\right) d \Gamma=L(t)\left(\mathbf{v}^{h}\right), \\
\mathbf{u}^{h}(0, \cdot)=\mathbf{u}_{0}^{h}, \quad \dot{\mathbf{u}}^{h}(0, \cdot)=\dot{\mathbf{u}}_{0}^{h},
\end{array}\right.
$$

where $\mathbf{u}_{0}^{h}$ (resp. $\dot{\mathbf{u}}_{0}^{h}$ ) is an approximation in $\mathbf{V}^{h}$ of the initial displacement $\mathbf{u}_{0}$ (resp. the initial velocity $\dot{\mathbf{u}}_{0}$ ). The notation $\langle\cdot, \cdot\rangle$ stands for the $L^{2}(\Omega)$ inner product.

\section{Existence and well-posedness results}

The proofs of this section are detailed in [10].

Theorem 2. Existence of discrete solutions for the static problem Let us suppose that $\gamma_{0}$ is small enough. Then for every $\Theta \in \mathbb{R}$ and $h>0$, the static problem (5) admits at least one solution. Moreover this solution satisfies the bound

$$
\left\|\mathbf{u}^{h}\right\|_{1, \Omega} \leq C
$$

where the constant $C>0$ depends only of the constants of $\mathbf{V}$-ellipticity of $a(\cdot, \cdot)$ and of continuity of $L(\cdot)$, but not on the friction coefficient $\mathscr{F}$ and on the Nitsche's parameter $\gamma_{0}$. 
Sketch of the proof: We introduce the auxillary problem involving (Tresca) friction $\mathcal{P}(g)$ with a fixed threshold $g \in L^{2}\left(\Gamma_{C}\right)$, and discretized with Nitsche:

$$
\mathcal{P}(g)\left\{\begin{array}{l}
\text { Find } \mathbf{u}^{h} \in \mathbf{V}^{h} \text { such that : } \\
A_{\Theta \gamma}\left(\mathbf{u}^{h}, \mathbf{v}^{h}\right)+\int_{\Gamma_{C}} \frac{1}{\gamma}\left[\mathrm{P}_{1, \gamma}^{\mathbf{n}}\left(\mathbf{u}^{h}\right)\right]_{\mathbb{R}^{-}} \mathbf{P}_{\Theta, \gamma}^{\mathbf{n}}\left(\mathbf{v}^{h}\right) d \Gamma \\
+\int_{\Gamma_{C}} \frac{1}{\gamma}\left[\mathbf{P}_{1, \gamma}^{\mathbf{t}}\left(\mathbf{u}^{h}\right)\right]_{g} \cdot \mathbf{P}_{\Theta, \gamma}^{\mathbf{t}}\left(\mathbf{v}^{h}\right) d \Gamma=L\left(\mathbf{v}^{h}\right), \quad \forall \mathbf{v}^{h} \in \mathbf{V}^{h} .
\end{array}\right.
$$

The solutions to Coulomb discrete problem (5) are the fixed point of the application $\phi^{h}: \mathbf{V}^{h} \rightarrow \mathbf{V}^{h}$ defined as follows: $\mathbf{w}^{h} \mapsto \mathbf{u}^{h}\left(\mathbf{w}^{h}\right)$ where $\mathbf{u}^{h}\left(\mathbf{w}^{h}\right)$ is the solution to $\mathcal{P}\left(\frac{-\mathscr{F}}{\gamma}\left[\mathrm{P}_{1, \gamma}^{\mathbf{n}}\left(\mathbf{w}^{h}\right)\right]_{\mathbb{R}^{-}}\right)$.

The application $\phi^{h}$ is well defined [4]. Using standard bounds and arguments, we show that $\phi^{h}$ is bounded and continuous in $\left(\mathbf{V}^{h},\|\cdot\|_{1, \Omega}\right)$. Thus we apply Brouwer's fixed point theorem to prove the existence of, at least, one solution to Problem (5).

\section{Proposition 3. Well-posedness:}

Static case : 1 . If $0 \leq \mathscr{F}<1$, assume there exists $C$ such that

$$
\mathscr{F} \leq C \frac{h}{\gamma_{0}}, \quad \frac{\left((1+\Theta)^{2}+\mathscr{F}\left(1+\Theta^{2}\right)\right)}{\gamma_{0}} \leq C .
$$

2. or if $\mathscr{F} \geq 1$, assume there exists $C$ such that

$$
\frac{\left(1+\Theta^{2}\right)\left(1+4 \mathscr{F}^{2}\right)}{\gamma_{0}} \leq C, \quad \mathscr{F} \leq C\left(\frac{h}{\gamma_{0}}\right)^{\frac{1}{2}},
$$

then Problem (5) admits one unique solution.

\section{Semi discretized dynamic case:}

For every value of $\Theta \in \mathbb{R}$ and $\gamma_{0}>0$, Problem (6) admits one unique solution $\mathbf{u}^{h} \in \mathscr{C}^{2}\left([0, T], \mathbf{V}^{h}\right)$.

Sketch of the proof: We introduce the following mesh- and parameterdependent scalar product in $\mathbf{V}^{h}$ :

$$
\left(\mathbf{v}^{h}, \mathbf{w}^{h}\right)_{\gamma}=\left(\mathbf{v}^{h}, \mathbf{w}^{h}\right)_{1, \Omega}+\left(\gamma^{-\frac{1}{2}} v_{n}^{h}, \gamma^{-\frac{1}{2}} w_{n}^{h}\right)_{0, \Gamma_{C}}+\left(\gamma^{-\frac{1}{2}} \mathbf{v}_{\mathbf{t}}^{h}, \gamma^{-\frac{1}{2}} \mathbf{w}_{\mathbf{t}}^{h}\right)_{0, \Gamma_{C}} .
$$

- Static case: we define the (non-linear) operator $\mathbf{B}^{h}: \mathbf{V}^{h} \rightarrow \mathbf{V}^{h}$ :

$$
\begin{aligned}
\left(\mathbf{B}^{h} \mathbf{v}^{h}, \mathbf{w}^{h}\right)_{\gamma}= & A_{\Theta \gamma}\left(\mathbf{v}^{h}, \mathbf{w}^{h}\right)+\int_{\Gamma_{C}} \frac{1}{\gamma}\left[\mathrm{P}_{1, \gamma}^{\mathbf{n}}\left(\mathbf{v}^{h}\right)\right]_{\mathbb{R}^{-}} \mathrm{P}_{\Theta, \gamma}^{\mathbf{n}}\left(\mathbf{w}^{h}\right) d \Gamma \\
& +\int_{\Gamma_{C}} \frac{1}{\gamma}\left[\mathbf{P}_{1, \gamma}^{\mathbf{t}}\left(\mathbf{v}^{h}\right)\right]\left(-\mathscr{F}\left[\mathrm{P}_{1, \gamma}^{\mathbf{n}}\left(\mathbf{u}^{h}\right)\right]_{\mathbb{R}^{-}}\right) \cdot \mathbf{P}_{\Theta, \gamma}^{\mathbf{t}}\left(\mathbf{w}^{h}\right) d \Gamma
\end{aligned}
$$


for all $\mathbf{v}^{h}, \mathbf{w}^{h} \in \mathbf{V}^{h}$. Then we prove that $\mathbf{B}^{h}$ is a one-to-one operator using Brezis' theorem [3].

- Semi discretized dynamic case: Nitsche's formulation leads to a system of (non-linear) second-order differential equations

$$
\left\{\begin{array}{l}
\text { Find } \mathbf{u}^{h}:[0, T] \rightarrow \mathbf{V}^{h} \text { such that for } t \in[0, T]: \\
\mathbf{M}^{h} \ddot{\mathbf{u}}^{h}(t)+\mathbf{B}^{h}\left(\mathbf{u}^{h}(t), \dot{\mathbf{u}}^{h}(t)\right)=\mathrm{E}^{h}(t), \\
\mathbf{u}^{h}(0, \cdot)=\mathbf{u}_{0}^{h}, \quad \dot{\mathbf{u}}^{h}(0, \cdot)=\dot{\mathbf{u}}_{0}^{h} .
\end{array}\right.
$$

with $\left(\mathrm{E}^{h}(t), \mathbf{w}^{h}\right)_{\gamma}=L(t)\left(\mathbf{w}^{h}\right)$, the mass operator $\mathbf{M}^{h}: \mathbf{V}^{h} \rightarrow \mathbf{V}^{h}$ defined by $\left(\mathbf{M}^{h} \mathbf{v}^{h}, \mathbf{w}^{h}\right)_{\gamma}=\left\langle\rho \mathbf{v}^{h}, \mathbf{w}^{h}\right\rangle$ and with $\mathbf{B}^{h}:\left(\mathbf{V}^{h}\right)^{2} \rightarrow \mathbf{V}^{h}$,

$$
\begin{aligned}
&\left(\mathbf{B}^{h}\left(\mathbf{v}^{h}, \dot{\mathbf{v}}^{h}\right), \mathbf{w}^{h}\right)_{\gamma}=A_{\Theta \gamma}\left(\mathbf{v}^{h}, \mathbf{w}^{h}\right)+\int_{\Gamma_{C}} \frac{1}{\gamma}\left[\mathrm{P}_{1, \gamma}^{\mathbf{n}}\left(\mathbf{v}^{h}\right)\right]_{\mathbb{R}^{-}} \mathrm{P}_{\Theta, \gamma}^{\mathbf{n}}\left(\mathbf{w}^{h}\right) d \Gamma \\
&+\int_{\Gamma_{C}} \frac{1}{\gamma}\left[Q_{\gamma}^{\mathbf{t}}\left(\mathbf{v}^{h}, \dot{\mathbf{v}}^{h}\right)\right]\left(-\mathscr{F}\left[\mathrm{P}_{1, \gamma}^{\mathbf{n}}\left(\mathbf{u}^{h}\right)\right]_{\mathbb{R}^{-}}\right) \\
& \cdot \mathbf{P}_{\Theta, \gamma}^{\mathbf{t}}\left(\mathbf{w}^{h}\right) d \Gamma,
\end{aligned}
$$

The operator $\mathbf{B}^{h}$ is Lipschitz-continuous and we conclude with the CauchyLipschitz theorem.

\section{$3 \quad$ Numerical results}

In what follows, we study an example where the three different zones characterizing friction (stick, slip and separation) exist. We consider the geometry $\hat{\Omega}=] 0,2[\times] 0,1\left[\right.$ and we adopt symmetry conditions (i.e., $u_{n}=0, \sigma_{t}(\mathbf{u})=$ $0)$ on $\left.\Gamma_{S}=\{1\} \times\right] 0,1[$. We achieve the computations on the square $\Omega=$ ] $0,1[\times] 0,1\left[\right.$. We set $\left.\Gamma_{C}=\right] 0,1\left[\times\{0\}\right.$ and $\Gamma_{N}=(] 0,1[\times\{1\}) \cup(\{0\} \times] 0,1[)$. We suppose that the body is homogeneous isotropic material and a Poisson ratio of $\nu=0.2$, a Young modulus of $E=10^{4}$ and a friction coefficient $\mathscr{F}=0.5$ are chosen. A density of surfaces forces $\mathrm{F}$ of magnitude $(0.5-y, 0)$ is applied on $\{0\} \times] 0.5,1[$ and one of magnitude $(0, x-0.5)$ is applied on ] $0.5,1\left[\times\{1\}\right.$. The Nitsche parameter $\gamma_{0}$ is fixed to $100 E$ and we consider the skew-symmetric case $\Theta=-1$. We achieve the numerical implementation with uniform meshes with the open source finite element library GetFEM++ (see http://getfem.org/download.html).

The solution for mesh sizes $h=\left[\frac{1}{4}, \frac{1}{8}, \frac{1}{16}, \frac{1}{32}, \frac{1}{64}\right]$ are compared with a reference solution on a very fine mesh $(h=1 / 128)$ and $P_{2}$ Lagrange elements. Moreover, the reference solution is computed with a different discretization of the friction problem (Lagrange multipliers and Alart-Curnier augmented 
lagrangian). Figure 1 depicts the Von Mises stress and we can note a transition point on $\Gamma_{C}$ between a contact part and a separation part. The Figure 2 shows the rates of convergence for the $H^{1}$ and $L^{2}$ relative norms with $P_{1}$ finite elements. For the $H^{1}$ norm we obtain the quasi optimality of the convergence rate whereas the suboptimality of the $L^{2}$ norm may come frim the lack of adjoint consistency when $\Theta=-1$.
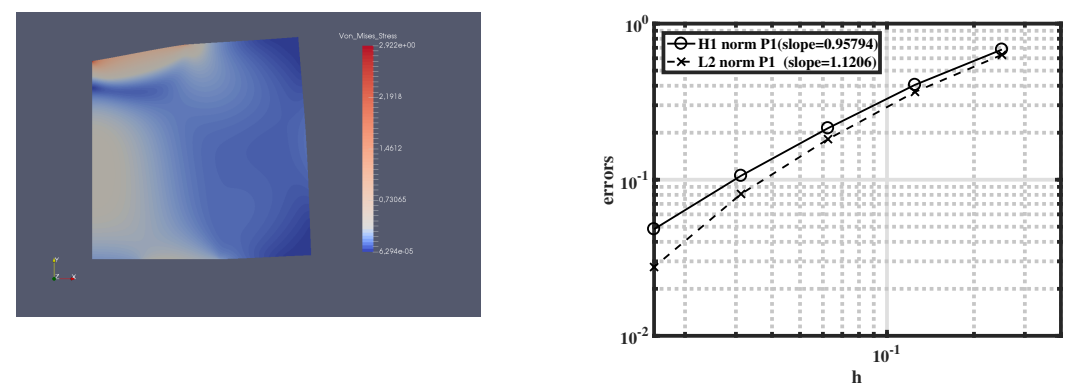

Fig. 1: Von Mises stress with displace- Fig. 2: $H^{1}$ and $L^{2}$ norms on the displacement amplified by 2000 ment $u_{h}$ for $P_{1}$ finite elements

\section{References}

1. P. Ballard And S. BAsseville, Existence and uniqueness for dynamical unilateral contact with Coulomb friction: a model problem, M2AN Math. Model. Numer. Anal. 39 (2005), 59-77.

2. A. Charles AND P. BAllard, Existence and uniqueness of solutions to dynamical unilateral contact problems with Coulomb friction: the case of a collection of points, ESAIM Math. Model. Numer. Anal. 48 (2014), 1-25.

3. H. BREZIS, Équations et inéquations non linéaires dans les espaces vectoriels en dualité, Ann. Inst. Fourier (Grenoble), 18 (1968), fasc. 1, 115-175.

4. F. Chouly, An adaptation of Nitsche's method to the Tresca friction problem, J. Math. Anal. Appl. 411 (2014), 329-339.

5. F. Chouly, M. Fabre, P. Hild, R. Mlika, J. Pousin and Y. Renard, An overview of recent results on Nitsche's method for contact problems, Lect. Notes Comput. Sci. Eng. To appear.

6. F. Chouly And P. Hild, A Nitsche-based method for unilateral contact problems: numerical analysis, SIAM J. Numer. Anal. 51 (2013), 1295-1307.

7. F. Chouly, P. Hild, And Y. Renard, A Nitsche finite element method for dynamic contact: 1 . Space semi-discretization and time-marching schemes, ESAIM Math. Model. Numer. Anal. 49 (2015), 481-502. 
8. _ _ A Nitsche finite element method for dynamic contact: 2. Stability of the schemes and numerical experiments, ESAIM Math. Model. Numer. Anal. 49 (2015), 503-528.

9. _ Symmetric and non-symmetric variants of Nitsche's method for contact problems in elasticity: theory and numerical experiments, Math. Comp. 84 (2015), 1089-1112.

10. F. Chouly, P. Hild, V. Lleras and Y. Renard, A Nitsche-based finite element method for contact with Coulomb friction, in preparation.

11. F. Chouly, R. Mlika, And Y. Renard, An unbiased Nitsche's approximation of the frictional contact between two elastic structures, Numerische Mathematik, To appear.

12. D. Doyen AND A. ERn, Analysis of the modified mass method for the dynamic Signorini problem with Coulomb friction, SIAM J. Numer. Anal. 49 (2011), 2039-2056.

13. C. ECK AND J. JARUSEK, Existence results for the static contact problem with coulomb friction, Math. Models Meth. Appl. Sci. 8 (1998), 445-468.

14. P. Hild AND Y. RENARD, Local uniqueness and continuation of solutions for the discrete Coulomb friction problem in elastostatics, Quart. Appl. Math. 63 (2005), 553-573.

15. T. Ligursky And Y. RenARD, A well-posed semi-discretization of elastodynamic contact problems with friction, Quart. J. Mech. Appl. Math. 64 (2011), $215-238$.

16. J. A. C. MARtins AND J. T. ODEN, Existence and uniqueness results for dynamic contact problems with nonlinear normal and friction interface laws, Nonlinear Anal. 11 (1987), 407-428.

17. J. Nitsche, Uber ein Variationsprinzip zur Lösung von Dirichlet-Problemen bei Verwendung von Teilräumen, die keinen Randbedingungen unterworfen sind, Abhandlungen aus dem Mathematischen Seminar der Universität Hamburg 36 (1971), 9-15.

18. R. Mlika, Y. Renard and F. Chouly, An unbiased Nitsche's formulation of large deformation frictional contact and self-contact, Comput. Methods Appl. Mech. Engrg., 325 (2017), 265-288.

19. Y. RENARD, A uniqueness criterion for the Signorini problem with Coulomb friction, SIAM J. Math. Anal. 38 (2006), 452-467.

20. Y. RENARD, Generalized Newton's methods for the approximation and resolution of frictional contact problems in elasticity, Comput. Methods Appl. Mech. Engrg., 256 (2013), 38-55. 